\title{
Tracking Control Design for Positive T-S Fuzzy Systems Under $H_{\infty}$ Performance
}

\author{
Lining FU a , Fucai LIU ${ }^{a}$, Aiwen MENG ${ }^{\text {a, }}{ }^{\text {, H.K. LAM }}{ }^{\mathrm{b}}$ and Jilong XU ${ }^{\mathrm{a}}$ \\ ${ }^{a}$ Institute of Electrical Engineering, Yanshan University, Qinghuangdao, Hebei, China \\ ${ }^{\mathrm{b}}$ Department of Informatics, King's College London, London, WC2R 2LS, UK
}

\begin{abstract}
This paper is dedicated to study the tracking control problem of the positive T-S Fuzzy systems under $H_{\infty}$ performance. Compared with general systems, the limitation of the positive systems will bring non-convex conditions into the stability analysis. In order to overcome the challenge brought by the transformation of non-convex conditions into convex conditions, a feasible solution is presented. In addition, by using the piecewise-linear membership functions (PLMFs) technology, more effective information of membership functions (MFs) is introduced, which effectively weakens the conservativeness of the stability conditions and achieves satisfactory tracking results. Ultimately, a simulation example is shown to confirm the reliability of the design method.
\end{abstract}

Keywords. Positive T-S Fuzzy Systems, $H_{\infty}$ Performance, Tracking Control, PLMFs

\section{Introduction}

Positive systems are ubiquitous in the real world, such as social demographic, network communication systems, etc [1]. These systems have the inherent property that their state variables are always positive under non-negative initial conditions [2]. Due to the limitation of the positivity, when positive conditions and stability conditions exist at the same time, the generation of non-convex conditions is inevitable [3], which will make the analysis of positive nonlinear systems more complicated and scarce. Therefore, the study of positive nonlinear systems has both practical value and challenges in theory.

Among all the nonlinear control strategies, the T-S fuzzy-model-based (TSFMB) control has attracted the attention of more and more scholars [4]-[6]. Therefore, this paper naturally uses the TSFMB to analyze the tracking control issue of positive systems. Although the application of tracking control can be seen everywhere [7]-[9], such as missile trajectory control [10] and aircraft attitude tracking [11], the existing literature on tracking control of positive systems is quite lacking. Thus, starting from the actual needs, the research on tracking control of positive systems have aroused our attention. In the existing literature, due to the insufficient introduction of the original membership function (MFs) information, the results achieved are extremely conservative. In this paper, more effective information of MFs is brought into the analysis condition, which is

\footnotetext{
${ }^{1}$ Corresponding Author: Meng Aiwen, The Key Laboratory of Industrial Computer Control Engineering of Hebei Province, Yanshan University, Qinhuangdao, Hebei 066004, China; E-mail: 1309494495@qq.com.
} 
helpful to realize a satisfactory tracking effect. In summary, the research motivation of the tracking control of the positive system is mature.

In order to distinguish from the previous work, the main contribution of this paper is to successfully extend the research of tracking control from general systems to positive systems that are rarely discussed. Correspondingly, for dealing with the non-convex problem generated by positive systems, this paper presents an effective approach so that feasible solutions can be found. In addition, for the purpose of achieving better tracking effect, this paper uses PLMFs technology to introduce more effective MFs information into the analysis conditions.

The following notations will refer to the full paper. $\delta_{r s}$ represents the $r$-th row and $s$-th column of the matrix $\Delta \in \mathfrak{R}^{n \times m} . \Delta \prec 0, \Delta \succ 0, \Delta \preceq 0$ and $\Delta \succeq 0$ represent that each element $\delta_{r s}$ is negative, positive, non-positive and non-negative, respectively. The Metzler matrix means that its off diagonal elements are all non-negative. $\breve{d}$ represents $\breve{d}=\{1,2, \ldots, d\}$.

\section{Preliminaries}

\subsection{Positive T-S Fuzzy Model}

The dynamic behavior of nonlinear plants is described by using $p$ rules, where the $i$-th rule is as follows:

$$
\begin{aligned}
& \text { Rule } i, i \in \breve{p}: \operatorname{IF} \varphi_{1}(\mathbf{x}(t)) \text { is } M_{1}^{i} \text { AND } \cdots \text { AND } \varphi_{\psi}(\mathbf{x}(t)) \text { is } M_{\psi}^{i} \\
& \text { THEN } \dot{\mathbf{x}}(t)=\mathbf{A}_{i} \mathbf{x}(t)+\mathbf{B}_{i} \mathbf{u}(t), \mathbf{x}(t)=\boldsymbol{\phi}(t),
\end{aligned}
$$

where $M_{l}^{i}$ is the fuzzy set, $\varphi_{l}(\mathbf{x}(t))$ is the premise variable, $l \in \breve{\psi}, \boldsymbol{\phi}(t)$ is the vector valued initial function. $\mathbf{A}_{i}$ and $\mathbf{B}_{i}$ are the system matrices with matching dimensions.

The whole system dynamics is shown as follows:

$$
\dot{\mathbf{x}}(t)=\sum_{i=1}^{p} w_{i}(\mathbf{x}(t))\left(\mathbf{A}_{i} \mathbf{x}(t)+\mathbf{B}_{i} \mathbf{u}(t)\right),
$$

where $w_{i}(\mathbf{x}(t))=\prod_{l=1}^{\psi} \mu_{M_{l}^{i}}\left(\varphi_{l}(\mathbf{x}(t))\right) / \sum_{k=1}^{p} \prod_{l=1}^{\psi} \mu_{M_{l}^{k}}\left(\varphi_{l}(\mathbf{x}(t))\right) \cdot w_{i}(\mathbf{x}(t)) \in\left[\begin{array}{ll}0 & 1\end{array}\right]$ and $\sum_{i=1}^{p} w_{i}(\mathbf{x}(t))=1, \forall i$.

Definition 1 [12]: If the condition $\boldsymbol{\phi}(\cdot) \succcurlyeq 0$ satisfy corresponding $\mathbf{x}(t) \succcurlyeq 0, \forall t \geq 0$, then the system is regarded as a positive system.

Lemma 1 [13]: System (2) is positive if it satisfies the following: $\mathbf{A}_{i}$ is a Metzler matrix and $\mathbf{B}_{i} \succeq 0, \forall i$ when $\mathbf{u}(t) \succeq 0$.

\subsection{Positive Stable Reference Model}

The stable positive reference model is given:

$$
\dot{\mathbf{x}}_{r}(t)=\mathbf{A}_{r} \mathbf{x}_{r}(t)+\mathbf{B}_{r} \mathbf{r}(t),
$$


where $\mathbf{A}_{r} \in \mathfrak{R}^{n \times n}, \mathbf{B}_{r} \in \mathfrak{R}^{n \times m}, \mathbf{r}(t) \in \mathfrak{R}^{m \times 1}$ and $\mathbf{x}_{r}(t) \in \mathfrak{R}^{n \times 1}$. It is worth emphasizing that the reference model should be guaranteed to be stable.

\subsection{Fuzzy Controller Design}

By using $c$ rules to describe the T-S fuzzy controller, where the $j$-th rule is:

$$
\begin{aligned}
& \text { Rule } j, j \in \breve{c}: \text { IF } \vartheta_{1}(\mathbf{x}(t)) \text { is } N_{1}^{j} \text { AND } \cdots \text { AND } \vartheta_{\Omega}(\mathbf{x}(t)) \text { is } N_{\Omega}^{j} \\
& \text { THEN } \quad \mathbf{u}(t)=\mathbf{F}_{j} \mathbf{e}(t)+\mathbf{G}_{j} \mathbf{x}_{r}(t),
\end{aligned}
$$

where $\vartheta_{d}(\mathbf{x}(t))$ is the premise variable, $N_{d}^{j}$ is the fuzzy set, $\mathbf{e}(t)=\mathbf{x}(t)-\mathbf{x}_{r}(t) . \mathbf{F}_{j} \in \Re^{m \times n}$ and $\mathbf{G}_{j} \in \mathfrak{R}^{m \times n}$ are the feedback gains, $d \in \breve{\Omega}$.

The overall T-S fuzzy controller is displayed as follows:

$$
\mathbf{u}(t)=\sum_{j=1}^{c} m_{j}(\mathbf{x}(t))\left(\mathbf{F}_{j} \mathbf{e}(t)+\mathbf{G}_{j} \mathbf{x}_{r}(t)\right),
$$

where $m_{j}(\mathbf{x}(t))=\prod_{d=1}^{\Omega} \mu_{N_{d}^{j}}\left(\vartheta_{d}(\mathbf{x}(t))\right) / \sum_{k=1}^{c} \prod_{d=1}^{\Omega} \mu_{N_{d}^{k}}\left(\vartheta_{d}(\mathbf{x}(t))\right) . m_{j}(\mathbf{x}(t)) \in\left[\begin{array}{ll}0 & 1\end{array}\right]$, $\sum_{j=1}^{c} m_{j}(\mathbf{x}(t))=1, \forall j$, and $m_{j}(\mathbf{x}(t))$ is the normalized grade of membership. In the following analysis, if there is no confusion, the time $t$ in each variable will be omitted.

\section{Positivity and Stability Analysis}

Combining (2) and (5), the closed-loop system is given:

$$
\dot{\mathbf{x}}=\sum_{i=1}^{p} \sum_{j=1}^{c} w_{i} m_{j}\left(\left(\mathbf{A}_{i}+\mathbf{B}_{i} \mathbf{F}_{j}\right) \mathbf{x}+\mathbf{B}_{i}\left(\mathbf{G}_{j}-\mathbf{F}_{j}\right) \mathbf{x}_{r}\right) \text {. }
$$

In order to study the stability of the error system, $\dot{\mathbf{e}}$ can be obtained:

$$
\dot{\mathbf{e}}=\dot{\mathbf{x}}-\dot{\mathbf{x}}_{r}=\sum_{i=1}^{p} \sum_{j=1}^{c} w_{i} m_{j}\left(\mathbf{A}_{i}+\mathbf{B}_{i} \mathbf{F}_{j}\right) \mathbf{e}+\sum_{i=1}^{p} \sum_{j=1}^{c} w_{i} m_{j}\left(\mathbf{A}_{i}-\mathbf{A}_{r}+\mathbf{B}_{i} \mathbf{G}_{j}\right) \mathbf{x}_{r}-\mathbf{B}_{r} \mathbf{r}
$$

Since positive conditions and stability conditions are met at the same time, nonconvex conditions will arise. Thus, for coping with this problem, a diagonally positive definite matrix $\mathbf{X} \in \mathfrak{R}^{n \times n}$ is defined. Defining $\mathbf{F}_{j} \mathbf{X}=\mathbf{M}_{j}$ and $\mathbf{G}_{j} \mathbf{X}=\mathbf{N}_{j}$, according to Lemma 1, post-multiplying both sides of all positive conditions by $\mathbf{X}$ at the same time, then the positive conditions can be obtained, i.e., $\left(\mathbf{A}_{i}+\mathbf{B}_{i} \mathbf{F}_{j}\right) \mathbf{X}=\mathbf{A}_{i} \mathbf{X}+\mathbf{B}_{i} \mathbf{M}_{j}$ is Metzler matrix and $\left(\mathbf{B}_{i}\left(\mathbf{G}_{j}-\mathbf{F}_{j}\right)\right) \mathbf{X}=\mathbf{B}_{i}\left(\mathbf{N}_{j}-\mathbf{M}_{j}\right) \succeq 0, \forall i, j$.

For further dealing with the non-convex stability conditions, based on the previous definition, (7) will be analyzed as:

$$
\begin{aligned}
\dot{\mathbf{e}} & =\sum_{i=1}^{p} \sum_{j=1}^{c} w_{i} m_{j}\left(\mathbf{A}_{i} \mathbf{X}+\mathbf{B}_{i} \mathbf{M}_{j}\right) \mathbf{X}^{-1} \mathbf{e}+\sum_{i=1}^{p} \sum_{j=1}^{c} w_{i} m_{j}\left(\mathbf{A}_{i} \mathbf{X}-\mathbf{A}_{r} \mathbf{X}+\mathbf{B}_{i} \mathbf{N}_{j}\right) \mathbf{X}^{-1} \mathbf{x}_{r} \\
& -\mathbf{B}_{r} \mathbf{r}=\sum_{i=1}^{p} \sum_{j=1}^{c} w_{i} m_{j} \mathbf{\Psi}_{i j} \mathbf{Z},
\end{aligned}
$$


where $\boldsymbol{\Psi}_{i j}=\left[\boldsymbol{\Psi}_{i j}^{(1)} \boldsymbol{\Psi}_{i j}^{(2)} \boldsymbol{\Psi}_{i j}^{(3)}\right], \boldsymbol{\Psi}_{i j}^{(1)}=\mathbf{A}_{i} \mathbf{X}+\mathbf{B}_{i} \mathbf{M}_{j}, \boldsymbol{\Psi}_{i j}^{(2)}=\mathbf{A}_{i} \mathbf{X}-\mathbf{A}_{r} \mathbf{X}+\mathbf{B}_{i} \mathbf{N}_{j}, \boldsymbol{\Psi}_{i j}^{(3)}=$ $-\mathbf{B}_{r}$ and $\mathbf{Z}^{T}=\left[\mathbf{Z}_{1}^{T} \mathbf{Z}_{2}^{T} \mathbf{Z}_{3}^{T}\right], \mathbf{Z}_{1}=\mathbf{X}^{-1} \mathbf{e}, \mathbf{Z}_{2}=\mathbf{X}^{-1} \mathbf{x}_{r}, \mathbf{Z}_{3}=\mathbf{r}$.

The following Lyapunov function candidate were selected for stability analysis:

$$
V(t)=\mathbf{e}^{T}(t) \mathbf{X}^{-1} \mathbf{e}(t) .
$$

For facilitating the stability analysis, we define $\mathbf{e}_{1}=\left[\begin{array}{lll}\mathbf{I}_{n} & \mathbf{0} & \mathbf{0}\end{array}\right], \mathbf{e}_{2}=\left[\begin{array}{lll}\mathbf{0} & \mathbf{I}_{n} & \mathbf{0}\end{array}\right]$ and $\mathbf{e}_{3}=\left[\begin{array}{lll}\mathbf{0} & \mathbf{0} & \mathbf{I}_{m}\end{array}\right]$. At the same time, $\dot{V}(t)$ is derived as:

$$
\dot{V}(t)=\sum_{i=1}^{p} \sum_{j=1}^{c} w_{i} m_{j} \mathbf{Z}^{T}\left(\Psi_{i j}^{T} \mathbf{e}_{1}+\mathbf{e}_{1}^{T} \Psi_{i j}\right) \mathbf{Z}=\sum_{i=1}^{p} \sum_{j=1}^{c} w_{i} m_{j} \mathbf{Z}^{T} \boldsymbol{\Xi}_{i j} \mathbf{Z} .
$$

For checking the tracking effect, the $H_{\infty}$ performance is utilized. Meanwhile, the variable $\Theta$ will be defined: $\Theta=\dot{V}+\mathbf{Z}_{1}^{T} \mathbf{Z}_{1}-\rho_{1} \mathbf{Z}_{2}^{T} \mathbf{Z}_{2}-\rho_{2} \mathbf{Z}_{3}^{T} \mathbf{Z}_{3}$, where $\rho_{1}>0$ and $\rho_{2}>0$.

Considering $\Theta<0$, then $\dot{V}(t) \leq-\mathbf{Z}_{1}^{T} \mathbf{Z}_{1}+\rho_{1} \mathbf{Z}_{2}^{T} \mathbf{Z}_{2}+\rho_{2} \mathbf{Z}_{3}^{T} \mathbf{Z}_{3}$. Set $t_{f}$ as the terminal time, thus, the $H_{\infty}$ performance [14] is given:

$$
\frac{\int_{0}^{t_{f}} \mathbf{Z}_{1}^{T} \mathbf{Z}_{1} d t-V(0)}{\int_{0}^{t_{f}}\left(\rho_{1} \mathbf{Z}_{2}^{T} \mathbf{Z}_{2}+\rho_{2} \mathbf{Z}_{3}^{T} \mathbf{Z}_{3}\right) d t} \leq 1 .
$$

Remark 1 : In order to meet the $H_{\infty}$ performance, inequality $\Theta<0$ must be guaranteed. where $\Theta=\sum_{i=1}^{p} \sum_{j=1}^{c} w_{i} m_{j} \mathbf{Z}^{T} \boldsymbol{\Lambda}_{i j} \mathbf{Z}$,

$$
\boldsymbol{\Lambda}_{i j}=\boldsymbol{\Xi}_{i j}+\mathbf{e}_{1}^{T} \mathbf{e}_{1}-\rho_{1} \mathbf{e}_{2}^{T} \mathbf{e}_{2}-\rho_{2} \mathbf{e}_{3}^{T} \mathbf{e}_{3}
$$

\section{Relaxed Positivity and Stability Analysis by PLMFs}

To relax the analysis conditions, PLMFs, explained in detail in [15], will be used as a method to extract more effective information of MFs. This includes digging the boundary information of $w_{i}(\mathbf{x}), m_{j}(\mathbf{x})$ and $\Delta h_{i j}(\mathbf{x})$ respectively, so that the conservativeness of the results can be weakened.

$$
\underline{\gamma}_{i j} \leq \Delta h_{i j}(\mathbf{x}) \leq \bar{\gamma}_{i j}, 0 \leq \underline{\sigma}_{i} \leq w_{i}(\mathbf{x}) \leq \bar{\sigma}_{i} \leq 1,0 \leq \underline{\tau}_{j} \leq m_{j}(\mathbf{x}) \leq \bar{\tau}_{j} \leq 1, \forall i, j,
$$

where $h_{i j}(\mathbf{x})=w_{i}(\mathbf{x}) m_{j}(\mathbf{x}), \tilde{h}_{i j}(\mathbf{x})$ and $\Delta h_{i j}(\mathbf{x})=h_{i j}(\mathbf{x})-\tilde{h}_{i j}(\mathbf{x})$ are the approximation function and the error function, respectively. $\underline{\gamma}_{i j}, \bar{\gamma}_{i j}, \underline{\sigma}_{i}, \bar{\sigma}_{i}, \underline{\tau}_{j}$ and $\bar{\tau}_{j}$ respectively represent the lower and upper boundaries of the corresponding functions.

To relax the research conditions, the above-mentioned boundary information of MFs is introduced through the following slack matrices $0<\mathbf{P}_{i j}=\mathbf{P}_{i j}^{T}, 0<\mathbf{Q}_{i j}=\mathbf{Q}_{i j}^{T}$ and $0<\mathbf{R}_{i j}=\mathbf{R}_{i j}^{T}, \mathbf{R}_{i j} \geq\left(\boldsymbol{\Lambda}_{i j}-\boldsymbol{P}_{i j}+\boldsymbol{Q}_{i j}\right)$. Thus, $\Theta$ can be dealt with as:

$$
\begin{aligned}
\Theta & =\sum_{i=1}^{p} \sum_{j=1}^{c} w_{i} m_{j} \mathbf{Z}^{T} \boldsymbol{\Lambda}_{i j} \mathbf{Z} \leq \sum_{i=1}^{p} \sum_{j=1}^{c} \mathbf{Z}^{T}\left(\left(\tilde{h}_{i j}(\mathbf{x})+\underline{\gamma}_{i j}\right)\left(\boldsymbol{\Lambda}_{i j}-\boldsymbol{P}_{i j}+\boldsymbol{Q}_{i j}\right)\right. \\
& \left.+\left(\bar{\gamma}_{i j}-\underline{\gamma}_{i j}\right) \mathbf{R}_{i j}+\overline{\boldsymbol{\sigma}}_{i} \bar{\tau}_{j} \mathbf{P}_{i j}-\underline{\boldsymbol{\sigma}}_{i} \underline{\tau}_{j} \mathbf{Q}_{i j}\right) \mathbf{Z} .
\end{aligned}
$$


It can be seen from (14) that inequality $\Theta<0$ can be satisfied if $\sum_{i=1}^{p} \sum_{j=1}^{c}\left(\left(\tilde{h}_{i j}(\mathbf{x})+\right.\right.$ $\left.\left.\underline{\gamma}_{i j}\right)\left(\boldsymbol{\Lambda}_{i j}-\boldsymbol{P}_{i j}+\boldsymbol{Q}_{i j}\right)+\left(\bar{\gamma}_{i j}-\underline{\gamma}_{i j}\right) \mathbf{R}_{i j}+\bar{\sigma}_{i} \bar{\tau}_{j} \mathbf{P}_{i j}-\underline{\sigma}_{i} \underline{\tau}_{j} \mathbf{Q}_{i j}\right)<0$ holds. In a word, the tracking control analysis results of the positive T-S fuzzy systems are given by the following theorem.

Theorem 1 Through scalars $\rho_{1}>0, \rho_{2}>0$, the tracking control of the positive T-S fuzzy system is guaranteed under satisfying the $H_{\infty}$ performance (11), if there is a diagonal positive definite matrix $\mathbf{X} \in \Re^{n \times n}$, the feedback gains $\mathbf{M}_{j} \in \mathfrak{R}^{m \times n}, \mathbf{N}_{j} \in \mathfrak{R}^{m \times n}, j \in \breve{c}$, symmetric matrices of matching dimensions $\mathbf{P}_{i j}=\mathbf{P}_{i j}^{T}, \mathbf{Q}_{i j}=\mathbf{Q}_{i j}^{T}$ and $\mathbf{R}_{i j}=\mathbf{R}_{i j}^{T}$ such that the GEVP has the feasible solution:

Minimize $\rho_{1}+\rho_{2}$

Subject to

1) $\left.\rho_{1}>0, \quad \rho_{2}>0 ; \quad 2\right) \quad \xi^{T}\left(\mathbf{X}-\varepsilon_{1} \mathbf{I}\right) \boldsymbol{\xi}$ is $S O S$;

3) $a_{i r s} \mathbf{X}+b_{i r} \mathbf{M}_{j} \quad$ is SOS; $\left.\quad \forall r \neq s ; \quad 4\right) \boldsymbol{\xi}^{T}\left(\mathbf{b}_{i r}\left(\mathbf{N}_{j}-\mathbf{M}_{j}\right)-\varepsilon_{2} \mathbf{I}\right) \boldsymbol{\xi}$ is SOS;

5) $\boldsymbol{\xi}^{T}\left(\mathbf{P}_{i j}-\varepsilon_{3} \mathbf{I}\right) \boldsymbol{\xi}$ is $\left.\operatorname{SOS} ; \quad 6\right) \boldsymbol{\xi}^{T}\left(\mathbf{Q}_{i j}-\varepsilon_{3} \mathbf{I}\right) \boldsymbol{\xi}$ is $\left.S O S ; \quad 7\right) \boldsymbol{\xi}^{T}\left(\mathbf{R}_{i j}-\varepsilon_{3} \mathbf{I}\right) \boldsymbol{\xi}$ is SOS;

8) $\boldsymbol{\xi}^{T}\left(\mathbf{R}_{i j} \geq\left(\boldsymbol{\Lambda}_{i j}-\boldsymbol{P}_{i j}+\boldsymbol{Q}_{i j}\right)-\varepsilon_{3} \mathbf{I}\right) \boldsymbol{\xi}$ is SOS; 9$)-\boldsymbol{\xi}^{T}\left(\sum_{i=1}^{p} \sum_{j=1}^{c}\left(\left(\tilde{h}_{i j}(\mathbf{x})+\underline{\gamma}_{i j}\right)\right.\right.$

$$
\left.\left.\left(\boldsymbol{\Lambda}_{i j}-\boldsymbol{P}_{i j}+\boldsymbol{Q}_{i j}\right)+\left(\bar{\gamma}_{i j}-\underline{\gamma}_{i j}\right) \mathbf{R}_{i j}+\bar{\sigma}_{i} \bar{\tau}_{j} \mathbf{P}_{i j}-\underline{\sigma}_{i} \underline{\tau}_{j} \mathbf{Q}_{i j}\right)+\varepsilon_{4} \mathbf{I}\right) \boldsymbol{\xi} \text { is } S O S, \forall i, j
$$

where the controller gains can be obtained by $\mathbf{F}_{j}=\mathbf{M}_{j} \mathbf{X}^{-1}, \mathbf{G}_{j}=\mathbf{N}_{j} \mathbf{X}^{-1}$.

Remark 2 : When only sub-conditions 1) to 4) in Theorem 1 and $\boldsymbol{\Lambda}_{i j}<0$ in (12) are satisfied, the situation where PLMFs are not used will be obtained, that is, the information of MFs is not applied to the analysis conditions.

\section{Simulation Example}

A simulation example is presented to confirm the reliability of the controller design. Un$\operatorname{der} \mathbf{x}=\left[\begin{array}{ll}x_{1} & x_{2}\end{array}\right]^{T}$, three-rule positive T-S fuzzy model is shown as follows:

$$
\begin{aligned}
& \mathbf{A}_{1}=\left[\begin{array}{cc}
1.18 & 0.46 \\
6.72 & -13.63
\end{array}\right], \mathbf{A}_{2}=\left[\begin{array}{cc}
0.85 & 1.25 \\
4.59 & -12.6
\end{array}\right], \mathbf{A}_{3}=\left[\begin{array}{cc}
0.69 & 0.35 \\
7.21 & -8.86
\end{array}\right], \\
& \mathbf{B}_{1}=\left[\begin{array}{l}
3.63 \\
0.05
\end{array}\right], \mathbf{B}_{2}=\left[\begin{array}{l}
1.42 \\
0.12
\end{array}\right], \mathbf{B}_{3}=\left[\begin{array}{l}
2.85 \\
0.86
\end{array}\right] .
\end{aligned}
$$

The positive reference model is set to:

$$
\mathbf{A}_{r}=\left[\begin{array}{cc}
-3.5 & 1.06 \\
6.35 & -12.5
\end{array}\right], \quad \mathbf{B}_{r}=\left[\begin{array}{l}
1.26 \\
0.31
\end{array}\right], \mathbf{r}(t)=1+0.5 \sin (0.2 t)
$$




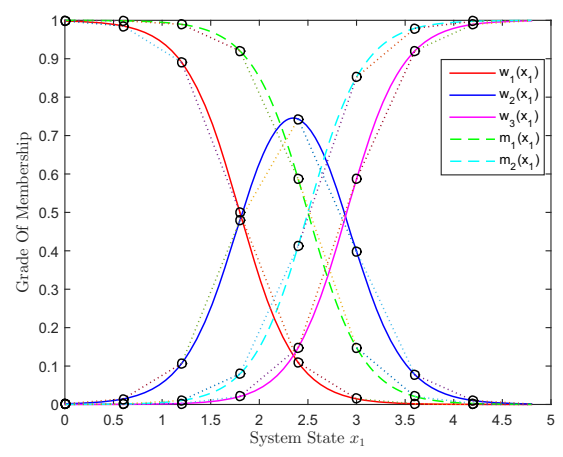

Figure 1. Positive fuzzy model: $w_{1}\left(x_{1}\right), w_{2}\left(x_{1}\right), w_{3}\left(x_{1}\right)$. Fuzzy controller: $m_{1}\left(x_{1}\right), m_{2}\left(x_{1}\right)$. The sampling points are set to $x_{1}(t)=\{0 ; 0.6 ; \cdots ; 4.8\}$.

The MFs of the positive fuzzy model and the controller are respectively set as: $w_{1}\left(x_{1}\right)=1-\frac{1}{1+e^{-3.5\left(x_{1}-1.8\right)}}, w_{2}\left(x_{1}\right)=1-w_{1}\left(x_{1}\right)-w_{3}\left(x_{1}\right), w_{3}\left(x_{1}\right)=\frac{1}{1+e^{-3.5\left(x_{1}-2.9\right)}}$, and $m_{1}\left(x_{1}\right)=1-\frac{1}{1+e^{-3.5\left(x_{1}-2.5\right)}}, m_{2}\left(x_{1}\right)=1-m_{1}\left(x_{1}\right)$, which are shown in Figure 1. Set the initial condition to $\mathbf{x}(0)=\left[\begin{array}{ll}0.3 & 0.5\end{array}\right]$ and $\mathbf{x}_{r}(0)=\left[\begin{array}{ll}0.3 & 0\end{array}\right]$, by using the designed controller, the state-time simulation results obtained under Remark 2 and Theorem 1 are presented in Figure 2 and Figure 3, respectively, which proves that the tracking effect is satisfactory.

By comparing Figure 2 and Figure 3, it can be concluded that the tracking effect of Figure 3 is significantly better, which proves the importance of MFs information introduced by the PLMFs method. It is worth mentioning that the more segments in the PLMFs method, the smaller the approximation error obtained, and the better the tracking effect obtained.
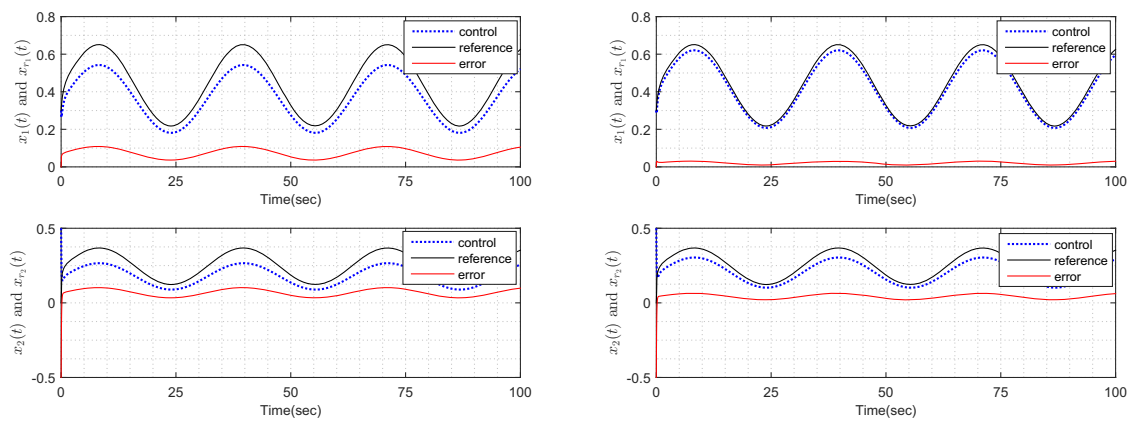

Figure 2. Tracking effect and tracking error Figure 3. Tracking effect and tracking error with $\rho_{1}=0.3111$ and $\rho_{2}=0.2086$. Top panel: with $\rho_{1}=0.4248$ and $\rho_{2}=0.1855$. Top panel: $e_{1 \max }=0.1087, R M S E 1=0.0778$. Bottom panel: $e_{1 \max }=0.0305, R M S E 1=0.0220$. Bottom panel: $e_{2 \max }=0.1021, R M S E 2=0.0860$. $e_{2 \max }=0.0640, R M S E 2=0.0647$. 


\section{Conclusion}

This paper is dedicated to the design of the tracking control of the positive T-S fuzzy system. To suppress tracking errors, a method of combining the MFs information with the $H_{\infty}$ performance is adopted. In addition, the non-convex problem due to the constraints of the positive system has been overcome. On the other hand, in order to weaken the conservativeness of the analysis results brought by the lack of MFs, the PLMFs method has been considered to introduce more effective information of MFs into the stability analysis. A simulation example has verified the reliability of the control strategy.

This work was supported by Natural Science Foundation of Hebei Province under Project Number F2019203505 and Kings College London.

\section{References}

[1] Y. Ebihara, D. Peaucelle and D. Arzelier, "Analysis and Synthesis of Interconnected Positive Systems," in IEEE Transactions on Automatic Control, vol. 62, no. 2, pp. 652-667, Feb. 2017, doi: 10.1109/TAC.2016.2558287.

[2] Forni F, Sepulchre R. Differentially positive systems[J]. Automatic Control IEEE Transactions on, 2014, 61(2):346-359.

[3] L. Fu, H.K.Lam, F.Liu, B.Xiao and Z.Zhong, "Static Output-Feedback Tracking Control For Positive Polynomial Fuzzy Systems," in IEEE Transactions on Fuzzy Systems, doi: 10.1109/TFUZZ.2021.3065521.

[4] Kang M. Structure identification of fuzzy model[J]. Fuzzy Sets and Systems, 1988.

[5] Ghorbal H , Hajjaji A E , Souissi M , et al. Robust Tracking Control for Takagi-Sugeno Systems With Unmeasurable Premise Variables: Application to Tank System[J]. Journal of Dynamic Systems Measurement Control, 2014, 136(4).

[6] S. Dong, C. L. P. Chen, M. Fang and Z. -G. Wu, ”Dissipativity-Based Asynchronous Fuzzy Sliding Mode Control for T-S Fuzzy Hidden Markov Jump Systems," in IEEE Transactions on Cybernetics, vol. 50, no. 9, pp. 4020-4030, Sept. 2020, doi: 10.1109/TCYB.2019.2919299.

[7] Y. Yu, H. Lam and K. Y. Chan, "T-S Fuzzy-Model-Based Output Feedback Tracking Control With Control Input Saturation," in IEEE Transactions on Fuzzy Systems, vol. 26, no. 6, pp. 3514-3523, Dec. 2018, doi: 10.1109/TFUZZ.2018.2835761.

[8] C. Zhang, H.K. Lam, J. Qiu, P. Qi and Q. Chen, "Fuzzy-Model-Based Output Feedback Steering Control in Autonomous Driving Subject to Actuator Constraints,' in IEEE Transactions on Fuzzy Systems, vol. 29, no. 3, pp. 457-470, March 2021, doi: 10.1109/TFUZZ.2019.2955044.

[9] Karimi, Hamid-Reza Rathinasamy, Sakthivel Palanisamy, Selvaraj. (2016). EID based repetitive tracking control for Takagi-Sugeno fuzzy systems with saturating actuator. IET Control Theory Applications. 10. 10.1049/iet-cta.2016.0036.

[10] Tseng C, Chen B S, Uang H J. Fuzzy tracking control design for nonlinear dynamic systems via T-S fuzzy model[J]. IEEE Transactions on Fuzzy Systems, 2001.

[11] Lee T, Leok M, M Cc Lamroch N H. Geometric Tracking Control of a Quadrotor UAV on SE(3)[J]. IEEE, 2010.

[12] Mao Y, Zhang H, Dang C. Stability Analysis and Constrained Control of a Class of Fuzzy Positive Systems with Delays Using Linear Copositive Lyapunov Functional[J]. Circuits Systems Signal Processing, 2012, 31(5):1863-1875.

[13] Li X, Lam H K, Ge S, et al. Stability Analysis of Positive Polynomial Fuzzy-Model-Based Control Systems with Time Delay under Imperfect Premise Matching[J]. IEEE Transactions on Fuzzy Systems, 2018, 26(4):2289-2300.

[14] Lam, H. K, Hongyi. Output-Feedback Tracking Control for Polynomial Fuzzy-Model-Based Control Systems.[J]. IEEE Transactions on Industrial Electronics, 2013.

[15] Zhao Y , Bo X, Liu C , et al. Relaxed LMI-based stability conditions for fuzzy-model-based control systems under imperfect premise matching: Approximated membership function approach[C]// Intelligent Control Automation. IEEE, 2015. 